Article

\title{
Initial Value Problem For Nonlinear Fractional Differential Equations With $\psi$-Caputo Derivative via Monotone Iterative Technique
}

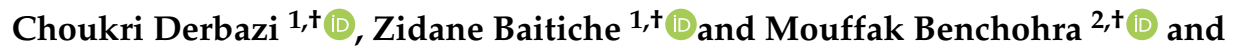 \\ Alberto Cabada $3, *,+$ (D) \\ 1 Laboratory of Mathematics and Applied Sciences, University of Ghardaia, Metlili 47000, Algeria; \\ choukriedp@yahoo.com (C.D.); baitichezidane19@gmail.com (Z.B.) \\ 2 Laboratory of Mathematics, Djillali Liabes University of Sidi-Bel-Abbes, Sidi Bel Abbès 22000, Algeria; \\ benchohra@yahoo.com \\ 3 Departamento de Estatistica, Análise Matemática e Optimización, Facultade de Matemáticas, \\ Instituto de Matemáticas, Universidade de Santiago de Compostela, 15705 Santiago de Compostela, Spain \\ * Correspondence: alberto.cabada@usc.gal \\ + These authors contributed equally to this work.
}

Received: 6 May 2020; Accepted: 16 May 2020; Published: 21 May 2020

check for updates

\begin{abstract}
In this article, we discuss the existence and uniqueness of extremal solutions for nonlinear initial value problems of fractional differential equations involving the $\psi$-Caputo derivative. Moreover, some uniqueness results are obtained. Our results rely on the standard tools of functional analysis. More precisely we apply the monotone iterative technique combined with the method of upper and lower solutions to establish sufficient conditions for existence as well as the uniqueness of extremal solutions to the initial value problem. An illustrative example is presented to point out the applicability of our main results.
\end{abstract}

Keywords: $\psi$-Caputo fractional derivative; Cauchy problem extremal solutions; monotone iterative technique; upper and lower solutions

\section{Introduction}

Fractional differential equations have been applied in many fields of engineering, physics, biology, and chemistry see [1-4]. Moreover, to get a couple of developments about the theory of fractional differential equations, one can allude to the monographs of Abbas et al. [5-7], Kilbas et al. [8], Miller and Ross [9], Podlubny [10], and Zhou [11,12], as well as to the papers by Agarwal, et al. [13], Benchohra, et al. [14-16], and the references therein. In the recent past, Almeida in [17] presented a new fractional differentiation operator called by $\psi$-Caputo fractional operator. For more details see [18-23], and the references given therein.

At the present day, different kinds of fixed point theorems are widely used as fundamental tools in order to prove the existence and uniqueness of solutions for various classes of nonlinear fractional differential equations for details, we refer the reader to a series of papers [24-30] and the references therein, but here we focus on those using the monotone iterative technique, coupled with the method of upper and lower solutions. This method is a very useful tool for proving the existence and approximation of solutions to many applied problems of nonlinear differential equations and integral equations (see [31-42]). However, as far as we know, there is no work yet reported on the existence of extremal solutions for the Cauchy problem with $\psi$-Caputo fractional derivative. Motivated 
by this fact, in this paper we deal with the existence and uniqueness of extremal solutions for the following initial value problem of fractional differential equations involving the $\psi$-Caputo derivative:

$$
\left\{\begin{array}{l}
{ }^{c} D_{a^{+}}^{\alpha ; \psi} x(t)=f(t, x(t)), t \in \mathrm{J}:=[a, b], \\
x(a)=a^{*},
\end{array}\right.
$$

where ${ }^{c} D_{a^{+}}^{\alpha ; \psi}$ is the $\psi$-Caputo fractional derivative of order $\alpha \in(0,1], f:[a, b] \times \mathbb{R} \longrightarrow \mathbb{R}$ is a given continuous function and $a^{*} \in \mathbb{R}$.

The rest of the paper is organized as follows: in Section 2, we give some necessary definitions and lemmas. The main results are given in Section 3. Finally, an example is presented to illustrate the applicability of the results developed.

\section{Preliminaries}

In this section, we introduce some notations and definitions of fractional calculus and present preliminary results needed in our proofs later.

We begin by defining $\psi$-Riemann-Liouville fractional integrals and derivatives. In what follows,

Definition $1([8,17])$. For $\alpha>0$, the left-sided $\psi$-Riemann-Liouville fractional integral of order $\alpha$ for an integrable function $x: \mathrm{J} \longrightarrow \mathbb{R}$ with respect to another function $\psi: \mathrm{J} \longrightarrow \mathbb{R}$ that is an increasing differentiable function such that $\psi^{\prime}(t) \neq 0$, for all $t \in \mathrm{J}$ is defined as follows

$$
I_{a^{+}}^{\alpha ; \psi} x(t)=\frac{1}{\Gamma(\alpha)} \int_{a}^{t} \psi^{\prime}(s)(\psi(t)-\psi(s))^{\alpha-1} x(s) \mathrm{ds},
$$

where $\Gamma$ is the classical Euler Gamma function.

Definition 2 ([17]). Let $n \in \mathbb{N}$ and let $\psi, x \in C^{n}(\mathrm{~J}, \mathbb{R})$ be two functions such that $\psi$ is increasing and $\psi^{\prime}(t) \neq 0$, for all $t \in \mathrm{J}$. The left-sided $\psi$-Riemann-Liouville fractional derivative of a function $x$ of order $\alpha$ is defined by

$$
\begin{aligned}
D_{a^{+}}^{\alpha ; \psi} x(t) & =\left(\frac{1}{\psi^{\prime}(t)} \frac{d}{d t}\right)^{n} I_{a^{+}}^{n-\alpha ; \psi} x(t) \\
& =\frac{1}{\Gamma(n-\alpha)}\left(\frac{1}{\psi^{\prime}(t)} \frac{d}{d t}\right)^{n} \int_{a}^{t} \psi^{\prime}(s)(\psi(t)-\psi(s))^{n-\alpha-1} x(s) \mathrm{ds}
\end{aligned}
$$

where $n=[\alpha]+1$.

Definition 3 ([17]). Let $n \in \mathbb{N}$ and let $\psi, x \in C^{n}(\mathrm{~J}, \mathbb{R})$ be two functions such that $\psi$ is increasing and $\psi^{\prime}(t) \neq 0$, for all $t \in \mathrm{J}$. The left-sided $\psi$-Caputo fractional derivative of $x$ of order $\alpha$ is defined by

$$
{ }^{c} D_{a^{+}}^{\alpha ; \psi} x(t)=I_{a^{+}}^{n-\alpha ; \psi}\left(\frac{1}{\psi^{\prime}(t)} \frac{d}{d t}\right)^{n} x(t),
$$

where $n=[\alpha]+1$ for $\alpha \notin \mathbb{N}, n=\alpha$ for $\alpha \in \mathbb{N}$.

To simplify notation, we will use the abbreviated symbol

$$
x_{\psi}^{[n]}(t)=\left(\frac{1}{\psi^{\prime}(t)} \frac{d}{d t}\right)^{n} x(t) .
$$


From the definition, it is clear that

$$
{ }^{c} D_{a^{+}}^{\alpha ; \psi} x(t)=\left\{\begin{array}{cc}
\int_{a}^{t} \frac{\psi^{\prime}(s)(\psi(t)-\psi(s))^{n-\alpha-1}}{\Gamma(n-\alpha)} x_{\psi}^{[n]}(s) \mathrm{ds} & , \text { if } \alpha \notin \mathbb{N}, \\
x_{\psi}^{[n]}(t) & , \text { if } \alpha \in \mathbb{N} .
\end{array}\right.
$$

We note that if $x \in C^{n}(\mathrm{~J}, \mathbb{R})$ the $\psi$-Caputo fractional derivative of order $\alpha$ of $x$ is determined as

$$
{ }^{c} D_{a^{+}}^{\alpha ; \psi} x(t)=D_{a^{+}}^{\alpha ; \psi}\left[x(t)-\sum_{k=0}^{n-1} \frac{x_{\psi}^{[k]}(a)}{k !}(\psi(t)-\psi(a))^{k}\right] .
$$

(see, for instance, [17], Theorem 3).

Lemma 1 ([20]). Let $\alpha, \beta>0$, and $x \in L^{1}(\mathrm{~J}, \mathbb{R})$. Then

$$
I_{a^{+}}^{\alpha ; \psi} I_{a^{+}}^{\beta ; \psi} x(t)=I_{a^{+}}^{\alpha+\beta ; \psi} x(t), \text { a.e. } t \in \mathrm{J} .
$$

In particular, if $x \in C(\mathrm{~J}, \mathbb{R})$, then $I_{a^{+}}^{\alpha ; \psi} I_{a^{+}}^{\beta ; \psi} x(t)=I_{a^{+}}^{\alpha+\beta ; \psi} x(t), t \in \mathrm{J}$.

Lemma 2 ([20]). Let $\alpha>0$, The following holds:

If $x \in C(\mathrm{~J}, \mathbb{R})$ then

$$
{ }^{c} D_{a^{+}}^{\alpha ; \psi} I_{a^{+}}^{\alpha ; \psi} x(t)=x(t), t \in \mathrm{J} .
$$

If $x \in C^{n}(\mathrm{~J}, \mathbb{R}), n-1<\alpha<n$. Then

$$
I_{a^{+}}^{\alpha ; \psi}{ }^{c} D_{a^{+}}^{\alpha ; \psi} x(t)=x(t)-\sum_{k=0}^{n-1} \frac{x_{\psi}^{[k]}(a)}{k !}[\psi(t)-\psi(a)]^{k}, \quad t \in \mathrm{J} .
$$

Lemma $3([8,20])$. Let $t>a, \alpha \geq 0$, and $\beta>0$. Then

- $I_{a^{+}}^{\alpha ; \psi}(\psi(t)-\psi(a))^{\beta-1}=\frac{\Gamma(\beta)}{\Gamma(\beta+\alpha)}(\psi(t)-\psi(a))^{\beta+\alpha-1}$,

- ${ }^{c} D_{a^{+}}^{\alpha ; \psi}(\psi(t)-\psi(a))^{\beta-1}=\frac{\Gamma(\beta)}{\Gamma(\beta-\alpha)}(\psi(t)-\psi(a))^{\beta-\alpha-1}$,

- ${ }^{c} D_{a^{+}}^{\alpha ; \psi}(\psi(t)-\psi(a))^{k}=0$, for all $k \in\{0, \ldots, n-1\}, n \in \mathbb{N}$.

Definition 4 ([43]). The one-parameter Mittag-Leffler function $\mathbb{E}_{\alpha}(\cdot)$, is defined as:

$$
\mathbb{E}_{\alpha}(z)=\sum_{k=0}^{\infty} \frac{z^{k}}{\Gamma(\alpha k+1)}, \quad(z \in \mathbb{R}, \alpha>0) .
$$

Definition 5 ([43]). The Two-parameter Mittag-Leffler function $\mathbb{E}_{\alpha, \beta}(\cdot)$, is defined as:

$$
\mathbb{E}_{\alpha, \beta}(z)=\sum_{k=0}^{\infty} \frac{z^{k}}{\Gamma(\alpha k+\beta)}, \alpha, \beta>0 \text { and } z \in \mathbb{R} \text {. }
$$

Theorem 1 (Weissinger's fixed point theorem [44]). Assume $(E, d)$ to be a non empty complete metric space and let $\beta_{j} \geq 0$ for every $j \in \mathbb{N}$ such that $\sum_{j=0}^{n-1} \beta_{j}$ converges. Furthermore, let the mapping $\mathbb{T}: E \rightarrow E$ satisfy the inequality

$$
d\left(\mathbb{T}^{j} u, \mathbb{T}^{j} v\right) \leq \beta_{j} d(u, v)
$$

for every $j \in \mathbb{N}$ and every $u, v \in E$. Then, $\mathbb{T}$ has a unique fixed point $u^{*}$. Moreover, for any $v_{0} \in E$, the sequence $\left\{\mathbb{T}^{j} v_{0}\right\}_{j=1}^{\infty}$ converges to this fixed point $u^{*}$. 


\section{Main Results}

Let us recall the definition and lemma of a solution for problem (1). First of all, we define what we mean by a solution for the boundary value problem (1).

Definition 6. A function $x \in C(\mathrm{~J}, \mathbb{R})$ is said to be a solution of Equation (1) if $x$ satisfies the equation ${ }^{c} D_{a^{+}}^{\alpha ; \psi} x(t)=f(t, x(t))$, for each $t \in \mathrm{J}$ and the condition

$$
x(a)=a^{*}
$$

For the existence of solutions for problem (1) we need the following lemma for a general linear equation of $\alpha>0$, that generalizes expression (3.1.34) in [8].

Lemma 4. For a given $h \in C(\mathrm{~J}, \mathbb{R})$ and $\alpha \in(n-1, n]$, with $n \in \mathbb{N}$, the linear fractional initial value problem

$$
\left\{\begin{array}{l}
{ }^{c} D_{a^{+}}^{\alpha ; \psi} x(t)+r x(t)=h(t), \quad t \in \mathrm{J}:=[a, b], \\
x_{\psi}^{[k]}(a)=a_{k}, \quad k=0, \ldots, n-1
\end{array}\right.
$$

has a unique solution given by

$$
\begin{aligned}
x(t)= & \sum_{k=0}^{n-1} \frac{a_{k}}{k !}[\psi(t)-\psi(a)]^{k}-r \mathcal{I}_{a^{+}}^{\alpha ; \psi} x(t)+\mathcal{I}_{a^{+}}^{\alpha ; \psi} h(t) \\
= & \sum_{k=0}^{n-1} \frac{a_{k}}{k !}[\psi(t)-\psi(a)]^{k}-\frac{r}{\Gamma(\alpha)} \int_{a}^{t} \psi^{\prime}(s)(\psi(t)-\psi(s))^{\alpha-1} x(s) \mathrm{d} s \\
& +\frac{1}{\Gamma(\alpha)} \int_{a}^{t} \psi^{\prime}(s)(\psi(t)-\psi(s))^{\alpha-1} h(s) \mathrm{d} s .
\end{aligned}
$$

Moreover, the explicit solution of the Volterra integral equation (6) can be represented by

$$
\begin{aligned}
x(t)= & \sum_{k=0}^{n-1} a_{k}[\psi(t)-\psi(a)]^{k} \mathbb{E}_{\alpha, k+1}\left(-r(\psi(t)-\psi(a))^{\alpha}\right) \\
& +\int_{a}^{t} \psi^{\prime}(s)(\psi(t)-\psi(s))^{\alpha-1} \mathbb{E}_{\alpha, \alpha}\left(-r(\psi(t)-\psi(a))^{\alpha}\right) h(s) \mathrm{d} s,
\end{aligned}
$$

where $\mathbb{E}_{\alpha, \beta}(\cdot)$ is the two-parametric Mittag-Leffer function defined in (4).

Proof. Since $\alpha \in(n-1, n]$, from Lemma 2 we know that the Cauchy problem (5) is equivalent to the following Volterra integral equation

$$
\begin{aligned}
x(t)= & \sum_{k=0}^{n-1} \frac{a_{k}}{k !}[\psi(t)-\psi(a)]^{k}-r \mathcal{I}_{a^{+}}^{\alpha ; \psi} x(t)+\mathcal{I}_{a^{+}}^{\alpha ; \psi} h(t) \\
= & \sum_{k=0}^{n-1} \frac{a_{k}}{k !}[\psi(t)-\psi(a)]^{k}-\frac{r}{\Gamma(\alpha)} \int_{a}^{t} \psi^{\prime}(s)(\psi(t)-\psi(s))^{\alpha-1} x(s) \mathrm{d} s \\
& +\frac{1}{\Gamma(\alpha)} \int_{a}^{t} \psi^{\prime}(s)(\psi(t)-\psi(s))^{\alpha-1} h(s) \mathrm{ds} .
\end{aligned}
$$

Note that the above equation can be written in the following form

$$
x(t)=\mathcal{T} x(t)
$$


where the operator $\mathcal{T}$ is defined by

$$
\mathcal{T} x(t)=\sum_{k=0}^{n-1} \frac{a_{k}}{k !}[\psi(t)-\psi(a)]^{k}-r \mathcal{I}_{a^{+}}^{\alpha ; \psi} x(t)+\mathcal{I}_{a^{+}}^{\alpha ; \psi} h(t) .
$$

Let $n \in \mathbb{N}$ and $x, y \in C(\mathrm{~J}, \mathbb{R})$. Then, we have

$$
\begin{aligned}
\left|\mathcal{T}^{n}(x)(t)-\mathcal{T}^{n}(y)(t)\right| & =\left|-r \mathcal{I}_{a^{+}}^{\alpha ; \psi}\left(\mathcal{T}^{n-1} x(t)-\mathcal{T}^{n-1} y(t)\right)\right| \\
& =\left|-r \mathcal{I}_{a^{+}}^{\alpha ; \psi}\left(-r \mathcal{I}_{a^{+}}^{\alpha ; \psi}\left(\mathcal{T}^{n-2} x(t)-\mathcal{T}^{n-2} y(t)\right)\right)\right| \\
& \vdots \\
& =\left|(-r)^{n} \mathcal{I}_{a^{+}}^{n ; \psi}(x(t)-y(t))\right| \\
& \leq \frac{\left(r(\psi(b)-\psi(a))^{\alpha}\right)^{n}}{\Gamma(n \alpha+1)}\|x-y\| .
\end{aligned}
$$

Hence, we have

$$
\left\|\mathcal{T}^{n}(x)-\mathcal{T}^{n}(y)\right\| \leq \frac{r^{n}(\psi(b)-\psi(a))^{n \alpha}}{\Gamma(n \alpha+1)}\|x-y\| .
$$

It's well known that

$$
\sum_{n=0}^{\infty} \frac{r^{n}(\psi(b)-\psi(a))^{n \alpha}}{\Gamma(n \alpha+1)}=\mathbb{E}_{\alpha}\left(r(\psi(b)-\psi(a))^{\alpha}\right),
$$

it follows that the mapping $\mathcal{T}^{n}$ is a contraction. Hence, by Weissinger's fixed point theorem, $\mathcal{T}$ has a unique fixed point. That is (5) has a unique solution.

Now we apply the method of successive approximations to prove that the integral Equation (6) can be expressed by

$$
\begin{aligned}
x(t) & =\sum_{k=0}^{n-1} a_{k}[\psi(t)-\psi(a)]^{k} \mathbb{E}_{\alpha, k+1}\left(-r(\psi(t)-\psi(a))^{\alpha}\right) \\
& +\int_{a}^{t} \psi^{\prime}(s)(\psi(t)-\psi(s))^{\alpha-1} \mathbb{E}_{\alpha, \alpha}\left(-r(\psi(t)-\psi(a))^{\alpha-1}\right) h(s) \mathrm{ds} .
\end{aligned}
$$

For this, we set

$$
\left\{\begin{aligned}
x_{0}(t)= & \sum_{k=0}^{n-1} \frac{a_{k}}{k !}[\psi(t)-\psi(a)]^{k} \\
x_{m}(t)= & x_{0}(t)-\frac{r}{\Gamma(\alpha)} \int_{a}^{t} \psi^{\prime}(s)(\psi(t)-\psi(s))^{\alpha-1} x_{m-1}(s) \mathrm{d} s \\
& +\frac{1}{\Gamma(\alpha)} \int_{a}^{t} \psi^{\prime}(s)(\psi(t)-\psi(s))^{\alpha-1} h(s) \mathrm{d} s .
\end{aligned}\right.
$$

It follows from Equation (8) and Lemma 3 that

$$
\begin{aligned}
x_{1}(t) & =x_{0}(t)-r \mathcal{I}_{a^{+}}^{\alpha ; \psi} x_{0}(t)+\mathcal{I}_{a^{+}}^{\alpha ; \psi} h(t) \\
& =\sum_{k=0}^{n-1} \frac{a_{k}}{k !}[\psi(t)-\psi(a)]^{k}-r \sum_{k=0}^{n-1} \frac{a_{k}}{\Gamma(\alpha+k+1)}[\psi(t)-\psi(a)]^{\alpha+k}+\mathcal{I}_{a^{+}}^{\alpha ; \psi} h(t) .
\end{aligned}
$$


Similarly, Equations (8) and (9) and Lemmas 1 and 3 yield

$$
\begin{aligned}
x_{2}(t)= & x_{0}(t)-r \mathcal{I}_{a^{+}}^{\alpha ; \psi} x_{1}(t)+\mathcal{I}_{a^{+}}^{\alpha ; \psi} h(t) \\
= & \sum_{k=0}^{n-1} \frac{a_{k}}{k !}[\psi(t)-\psi(a)]^{k}-r \mathcal{I}_{a^{+}}^{\alpha ; \psi}\left(\sum_{k=0}^{n-1} \frac{a_{k}}{k !}[\psi(t)-\psi(a)]^{k}\right. \\
& \left.-r \sum_{k=0}^{n-1} \frac{a_{k}}{\Gamma(\alpha+k+1)}[\psi(t)-\psi(a)]^{\alpha+k}+\mathcal{I}_{a^{+}}^{\alpha ; \psi} h(t)\right)+\mathcal{I}_{a^{+}}^{\alpha ; \psi} h(t) \\
= & \sum_{k=0}^{n-1} \frac{a_{k}}{k !}[\psi(t)-\psi(a)]^{k}-r \sum_{k=0}^{n-1} \frac{a_{k}}{\Gamma(\alpha+k+1)}[\psi(t)-\psi(a)]^{\alpha+k} \\
& +r^{2} \sum_{k=0}^{n-1} \frac{a_{k}}{\Gamma(2 \alpha+k+1)}[\psi(t)-\psi(a)]^{2 \alpha+k}-r \mathcal{I}_{a^{+}}^{2 \alpha ; \psi} h(t)+\mathcal{I}_{a^{+}}^{\alpha ; \psi} h(t) \\
= & \sum_{l=0}^{2} \sum_{k=0}^{n-1} \frac{(-r)^{l} a_{k}}{\Gamma(l \alpha+k+1)}[\psi(t)-\psi(a)]^{l \alpha+k}+\int_{a}^{t} \psi^{\prime}(s) \sum_{l=0}^{1} \frac{(-r)^{l-1}(\psi(t)-\psi(s))^{l \alpha+\alpha-1}}{\Gamma(l \alpha+\alpha)} h(s) \mathrm{ds} .
\end{aligned}
$$

Continuing this process, we derive the following relation

$$
x_{m}(t)=\sum_{l=0}^{m} \sum_{k=0}^{n-1} \frac{(-r)^{l} a_{k}}{\Gamma(l \alpha+k+1)}[\psi(t)-\psi(a)]^{l \alpha+k}+\int_{a}^{t} \psi^{\prime}(s) \sum_{l=0}^{m-1} \frac{(-r)^{l-1}(\psi(t)-\psi(s))^{l \alpha+\alpha-1}}{\Gamma(l \alpha+\alpha)} h(s) \mathrm{ds} .
$$

Taking the limit as $n \rightarrow \infty$, we obtain the following explicit solution $x(t)$ to the integral Equation (6):

$$
\begin{aligned}
x(t)= & \sum_{l=0}^{\infty} \sum_{k=0}^{n-1} \frac{(-r)^{l} a_{k}}{\Gamma(l \alpha+k+1)}[\psi(t)-\psi(a)]^{l \alpha+k}+\int_{a}^{t} \psi^{\prime}(s) \sum_{l=0}^{\infty} \frac{(-r)^{l-1}(\psi(t)-\psi(s))^{l \alpha+\alpha-1}}{\Gamma(l \alpha+\alpha)} h(s) \mathrm{ds} \\
= & \sum_{k=0}^{n-1} a_{k}(\psi(t)-\psi(a))^{k} \sum_{l=0}^{\infty} \frac{(-r)^{l}}{\Gamma(l \alpha+k+1)}[\psi(t)-\psi(a)]^{l \alpha} \\
& +\int_{a}^{t} \psi^{\prime}(s)(\psi(t)-\psi(s))^{\alpha-1} \sum_{l=0}^{\infty} \frac{(-r)^{l-1}(\psi(t)-\psi(s))^{l \alpha}}{\Gamma(l \alpha+\alpha)} h(s) \mathrm{ds} .
\end{aligned}
$$

Taking into account (4), we get

$$
\begin{aligned}
x(t) & =\sum_{k=0}^{n-1} a_{k}[\psi(t)-\psi(a)]^{k} \mathbb{E}_{\alpha, k+1}\left(-r(\psi(t)-\psi(a))^{\alpha}\right) \\
& +\int_{a}^{t} \psi^{\prime}(s)(\psi(t)-\psi(s))^{\alpha-1} \mathbb{E}_{\alpha, \alpha}\left(-r(\psi(t)-\psi(s))^{\alpha}\right) h(s) \mathrm{d} s .
\end{aligned}
$$

Then the proof is completed.

Lemma 5 (Comparison result). Let $\alpha \in(0,1]$ be fixed and $r \in \mathbb{R}$. If $\rho \in C(\mathrm{~J}, \mathbb{R})$ satisfies the following inequalities

$$
\left\{\begin{array}{l}
{ }^{c} D_{a^{+}}^{\alpha ; \psi} \rho(t) \geq-r \rho(t), \quad t \in[a, b] \\
\rho(a) \geq 0
\end{array}\right.
$$

then $\rho(t) \geq 0$ for all $t \in \mathrm{J}$.

Proof. Using the integral representation (7) and the fact that, $\mathbb{E}_{\alpha, 1}(z) \geq 0$ and $\mathbb{E}_{\alpha, \alpha}(z) \geq 0$ for all $\alpha \in(0,1]$ and $z \in \mathbb{R}$, (see [45]) it suffices to take $h(t)={ }^{c} \mathcal{D}_{a^{+}}^{\alpha ; \psi} \rho(t)+r \rho(t) \geq 0$ with initial conditions $\rho(a)=a^{*} \geq 0$. 
Definition 7. A function $x_{0} \in C(\mathrm{~J}, \mathbb{R})$ is said to be a lower solution of the problem (1), if it satisfies

$$
\left\{\begin{array}{l}
{ }^{c} D_{a^{+}}^{\alpha ; \psi} x_{0}(t) \leq f\left(t, x_{0}\right), \quad t \in(a, b], \\
x_{0}(a) \leq a^{*} .
\end{array}\right.
$$

Definition 8. A function $y_{0} \in C(\mathrm{~J}, \mathbb{R})$ is called an upper solution of problem (1), if it satisfies

$$
\left\{\begin{array}{l}
{ }^{c} D_{a^{+}}^{\alpha ; \psi} y_{0}(t) \geq f\left(t, y_{0}\right), \quad t \in(a, b] \\
y_{0}(a) \geq a^{*}
\end{array}\right.
$$

Theorem 2. Let the function $f \in C(\mathrm{~J} \times \mathbb{R}, \mathbb{R})$. In addition assume that:

$\left(H_{1}\right)$ There exist $x_{0}, y_{0} \in C(\mathrm{~J}, \mathbb{R})$ such that $x_{0}$ and $y_{0}$ are lower and upper solutions of problem (1), respectively, with $x_{0}(t) \leq y_{0}(t), t \in \mathrm{J}$.

$\left(H_{2}\right)$ There exists a constant $r \in \mathbb{R}$ such that

$$
f(t, y)-f(t, x) \geq-r(y-x) \text { for } x_{0} \leq x \leq y \leq y_{0} .
$$

Then there exist monotone iterative sequences $\left\{x_{n}\right\}$ and $\left\{y_{n}\right\}$, which converge uniformly on the interval $\mathrm{J}$ to the extremal solutions of $(1)$ in the sector $\left[x_{0}, y_{0}\right]$, where

$$
\left[x_{0}, y_{0}\right]=\left\{z \in C(\mathrm{~J}, \mathbb{R}): x_{0}(t) \leq z(t) \leq y_{0}(t), \quad t \in \mathrm{J}\right\} .
$$

Proof. First, for any $x_{0}(t), y_{0}(t) \in C(\mathrm{~J}, \mathbb{R})$, we consider the following linear initial value problems of fractional order:

$$
\left\{\begin{array}{l}
{ }^{c} D_{a^{+}}^{\alpha ; \psi} x_{n+1}(t)=f\left(t, x_{n}(t)\right)-r\left(x_{n+1}(t)-x_{n}(t)\right), \quad t \in \mathrm{J}, \\
x_{n+1}(a)=a^{*}
\end{array}\right.
$$

and

$$
\left\{\begin{array}{l}
{ }^{c} D_{a^{+}}^{\alpha ; \psi} y_{n+1}(t)=f\left(t, y_{n}(t)\right)-r\left(y_{n+1}(t)-y_{n}(t)\right), \quad t \in \mathrm{J}, \\
y_{n+1}(a)=a^{*} .
\end{array}\right.
$$

By Lemma 4, we know that (13) and (14) have unique solutions in $C(\mathrm{~J}, \mathbb{R})$ which are defined as follows:

$$
\begin{aligned}
& x_{n+1}(t)=a^{*} \mathbb{E}_{\alpha, 1}\left(-r(\psi(t)-\psi(a))^{\alpha}\right) \\
& +\int_{a}^{t} \psi^{\prime}(s)(\psi(t)-\psi(s))^{\alpha-1} \mathbb{E}_{\alpha, \alpha}\left(-r(\psi(t)-\psi(s))^{\alpha}\right)\left(f\left(s, x_{n}(s)\right)+r x_{n}(s)\right) \mathrm{d} s, t \in \mathrm{J}, \\
& y_{n+1}(t)=a^{*} \mathbb{E}_{\alpha, \alpha}\left(-r(\psi(t)-\psi(a))^{\alpha}\right) \\
& +\int_{a}^{t} \psi^{\prime}(s)(\psi(t)-\psi(s))^{\alpha-1} \mathbb{E}_{\alpha, \alpha}\left(-r(\psi(t)-\psi(s))^{\alpha}\right)\left(f\left(s, y_{n}(s)\right)+r y_{n}(s)\right) \mathrm{d} s, t \in \mathrm{J} .
\end{aligned}
$$

We will divide the proof into three steps.

Step 1: We show that the sequences $x_{n}(t), y_{n}(t)(n \geq 1)$ are lower and upper solutions of problem (1), respectively and the following relation holds

$$
x_{0}(t) \leq x_{1}(t) \leq \cdots \leq x_{n}(t) \leq \cdots \leq y_{n}(t) \leq \cdots \leq y_{1}(t) \leq y_{0}(t), \quad t \in \mathrm{J} .
$$


First, we prove that

$$
x_{0}(t) \leq x_{1}(t) \leq y_{1}(t) \leq y_{0}(t), \quad t \in \mathrm{J} .
$$

Set $\rho(t)=x_{1}(t)-x_{0}(t)$. From (13) and Definition 7, we obtain

$$
\begin{aligned}
{ }^{c} D_{a^{+}}^{\alpha ; \psi} \rho(t) & ={ }^{c} D_{a^{+}}^{\alpha ; \psi} x_{1}(t)-{ }^{c} D_{a^{+}}^{\alpha ; \psi} x_{0}(t) \\
& \geq f\left(t, x_{0}(t)\right)-r\left(x_{1}(t)-x_{0}(t)\right)-f\left(t, x_{0}(t)\right) \\
& =-r \rho(t) .
\end{aligned}
$$

Again, since

$$
\rho(a)=x_{1}(a)-x_{0}(a)=a^{*}-x_{0}(a) \geq 0 .
$$

By Lemma 5, $\rho(t) \geq 0$, for $t \in \mathrm{J}$. That is, $x_{0}(t) \leq x_{1}(t)$. Similarly, we can show that $y_{1}(t) \leq y_{0}(t), t \in \mathrm{J}$.

Now, let $\rho(t)=y_{1}(t)-x_{1}(t)$. From (13), (14) and (H2), we get

$$
\begin{aligned}
{ }^{c} D_{a^{+}}^{\alpha ; \psi} \rho(t) & ={ }^{c} D_{a^{+}}^{\alpha ; \psi} y_{1}(t)-{ }^{c} D_{a^{+}}^{\alpha ; \psi} x_{1}(t) \\
& =f\left(t, y_{0}(t)\right)-r\left(y_{1}(t)-y_{0}(t)\right)-f\left(t, x_{0}(t)\right)+r\left(x_{1}(t)-x_{0}(t)\right) \\
& =f\left(t, y_{0}(t)\right)-f\left(t, x_{0}(t)\right)-r\left(y_{1}(t)-y_{0}(t)\right)+r\left(x_{1}(t)-x_{0}(t)\right) \\
& \geq-r\left(y_{0}(t)-x_{0}(t)\right)-r\left(y_{1}(t)-y_{0}(t)\right)+r\left(x_{1}(t)-x_{0}(t)\right) \\
& =-r \rho(t) .
\end{aligned}
$$

Since, $\rho(a)=x_{1}(a)-y_{1}(a)=a^{*}-a^{*}=0$. By Lemma 5, we get $x_{1}(t) \leq y_{1}(t), t \in \mathrm{J}$.

Secondly, we show that $x_{1}(t), y_{1}(t)$ are lower and upper solutions of problem (1), respectively. Since $x_{0}$ and $y_{0}$ are lower and upper solutions of problem (1), by $\left(H_{2}\right)$, it follows that

$$
{ }^{c} D_{a^{+}}^{\alpha ; \psi} x_{1}(t)=f\left(t, x_{0}(t)\right)-r\left(x_{1}(t)-x_{0}(t)\right) \leq f\left(t, x_{1}(t)\right),
$$

also $x_{1}(a)=a^{*}$. Therefore, $x_{1}(t)$ is a lower solution of problem (1). Similarly, it can be obtained that $y_{1}(t)$ is an upper solution of problem (1).

By the above arguments and mathematical induction, we can show that the sequences $x_{n}(t), y_{n}(t),(n \geq 1)$ are lower and upper solutions of problem (1), respectively and the following relation holds

$$
x_{0}(t) \leq x_{1}(t) \leq \cdots \leq x_{n}(t) \leq \cdots \leq y_{n}(t) \leq \cdots \leq y_{1}(t) \leq y_{0}(t), \quad t \in \mathrm{J} .
$$

Step 2: The sequences $\left\{x_{n}(t)\right\},\left\{y_{n}(t)\right\}$ converge uniformly to their limit functions $x^{*}(t), y^{*}(t)$, respectively.

Note that the sequence $\left\{x_{n}(t)\right\}$ is monotone nondecreasing and is bounded from above by $y_{0}(t)$. Since the sequence $\left\{y_{n}(t)\right\}$ is monotone nonincreasing and is bounded from below by $x_{0}(t)$, therefore the pointwise limits exist and these limits are denoted by $x^{*}$ and $y^{*}$. Moreover, since $\left\{x_{n}(t)\right\},\left\{y_{n}(t)\right\}$ are sequences of continuous functions defined on the compact set $[a, b]$, hence by Dini's theorem [46], the convergence is uniform. This is

$$
\lim _{n \rightarrow \infty} x_{n}(t)=x^{*}(t) \quad \text { and } \quad \lim _{n \rightarrow \infty} y_{n}(t)=y^{*}(t),
$$

uniformly on $t \in \mathrm{J}$ and the limit functions $x^{*}, y^{*}$ satisfy problem (1). Furthermore, $x^{*}$ and $y^{*}$ satisfy the relation

$$
x_{0} \leq x_{1} \leq \cdots \leq x_{n} \leq x^{*} \leq y^{*} \leq \cdots \leq y_{n} \leq \cdots \leq y_{1} \leq y_{0} .
$$


Step 3: We prove that $x^{*}$ and $y^{*}$ are extremal solutions of problem (1) in $\left[x_{0}, y_{0}\right]$.

Let $z \in\left[x_{0}, y_{0}\right]$ be any solution of (1). We assume that the following relation holds for some $n \in \mathbb{N}$ :

$$
x_{n}(t) \leq z(t) \leq y_{n}(t), \quad t \in \mathrm{J} .
$$

Let $\rho(t)=z(t)-x_{n+1}(t)$. We have

$$
\begin{aligned}
{ }^{c} D_{a^{+}}^{\alpha ; \psi} \rho(t) & ={ }^{c} D_{a^{+}}^{\alpha ; \psi} z(t)-{ }^{c} D_{a^{+}}^{\alpha ; \psi} x_{n+1}(t) \\
& =f(t, z(t))-f\left(t, x_{n}(t)\right)+r\left(x_{n+1}(t)-x_{n}(t)\right) \\
& \geq-r\left(z(t)-x_{n}(t)\right)+r\left(x_{n+1}(t)-x_{n}(t)\right) \\
& =-r \rho(t) .
\end{aligned}
$$

Furthermore, $\rho(a)=z(a)-x_{n+1}(a)=a^{*}-a^{*}=0$. By Lemma 5, we obtain $\rho(t) \geq 0, t \in \mathrm{J}$, which means

$$
x_{n+1}(t) \leq z(t), t \in \mathrm{J} .
$$

Using the same method, we can show that

$$
z(t) \leq y_{n+1}(t), t \in \mathrm{J}
$$

Hence, we have

$$
x_{n+1}(t) \leq z(t) \leq y_{n+1}(t), t \in \mathrm{J} .
$$

Therefore, (19) holds on J for all $n \in \mathbb{N}$. Taking the limit as $n \rightarrow \infty$ on both sides of (19), we get

$$
x^{*} \leq z \leq y^{*} .
$$

Therefore $x^{*}, y^{*}$ are the extremal solutions of (1) in $\left[x_{0}, y_{0}\right]$. This completes the proof.

Now, we shall prove the uniqueness of the solution of the system (1) by monotone iterative technique.

Theorem 3. Suppose that (H1) and (H2) are satisfied. Furthermore, we impose that:

(H3) There exists a constant $r^{*} \geq-r$ such that

$$
f(t, y)-f(t, x) \leq r^{*}(y-x)
$$

for every $x_{0} \leq x \leq y \leq y_{0}, t \in \mathrm{J}$. Then problem (1) has a unique solution between $x_{0}$ and $y_{0}$.

Proof. From the Theorem 2, we know that $x^{*}(t)$ and $y^{*}(t)$ are the extremal solutions of the IVP (1) and $x^{*}(t) \leq y^{*}(t), t \in \mathrm{J}$. It is sufficient to prove $x^{*}(t) \geq y^{*}(t), t \in \mathrm{J}$. In fact, let $\rho(t)=x^{*}(t)-y^{*}(t), t \in \mathrm{J}$, in view of $(\mathrm{H} 3)$, we have

$$
\begin{aligned}
{ }^{c} D_{a^{+}}^{\alpha ; \psi} \rho(t) & ={ }^{c} D_{a^{+}}^{\alpha ; \psi} x^{*}(t)-{ }^{c} D_{a^{+}}^{\alpha ; \psi} y^{*}(t) \\
& =f\left(t, x^{*}(t)\right)-f\left(t, y^{*}(t)\right) \\
& \geq r^{*}\left(x^{*}(t)-y^{*}(t)\right) \\
& =r^{*} \rho(t) .
\end{aligned}
$$

Furthermore, $\rho(a)=x^{*}(a)-y^{*}(a)=a^{*}-a^{*}=0$. From Lemma 5, it follows that $\rho(t) \geq 0, t \in \mathrm{J}$. Hence, we obtain

$$
x^{*}(t) \geq y^{*}(t), \quad t \in \mathrm{J} .
$$

Therefore, $x^{*} \equiv y^{*}$ is the unique solution of the Cauchy problem (1) in $\left[x_{0}, y_{0}\right]$. This ends the proof of Theorem 3. 
As a direct consequence of the previous result, we arrive at the following one

Corollary 1. Suppose that (H1) is satisfied and that $f \in C(E, \mathbb{R})$, is differentiable with respect to $x$ and $\partial f / \partial x \in C(E, \mathbb{R})$, with

$$
E=\left\{(t, x) \in \mathbb{R}^{2}, \quad \text { such that } \quad x_{0}(t) \leq x \leq y_{0}(t)\right\}
$$

Then problem (1) has a unique solution between $x_{0}$ and $y_{0}$.

Proof. The proof follows immediately from the fact that $E$ is a compact set and, as a consequence, $\partial f / \partial x$ is bounded in $E$.

\section{An Example}

Example 1. Consider the following problem:

$$
\left\{\begin{array}{l}
{ }^{c} D_{0^{+}}^{\frac{1}{2}} x(t)=1-x^{2}(t)+2 t, \quad t \in \mathrm{J}:=[0,1] \\
x(0)=1
\end{array}\right.
$$

Note that, this problem is a particular case of IVP (1), where

$$
\alpha=\frac{1}{2}, a=0, b=1, a^{*}=1, \psi(t)=t,
$$

and $f: \mathrm{J} \times \mathbb{R} \longrightarrow \mathbb{R}$ given by

$$
f(t, x)=1-x^{2}+2 t, \quad \text { for } t \in \mathrm{J}, x \in \mathbb{R} .
$$

Taking $x_{0}(t) \equiv 0$ and $y_{0}(t)=1+t$, it is not difficult to verify that $x_{0}, y_{0}$ are lower and upper solutions of (20), respectively, and $x_{0} \leq y_{0}$. So $\left(H_{1}\right)$ of Theorem 2 holds

On the other hand, it is clear that the function $f$ is continuous and satisfies

$$
\left|\frac{f(t, x)}{\partial x}(t, x)\right|=|-2 x| \leq 4 \text { for all } t \in[0,1] \text { and } 0 \leq x \leq t+1 .
$$

Hence, by Corollary 1, the initial value problem (20) has a unique solution $u^{*}$ and there exist monotone iterative sequences $\left\{x_{n}\right\}$ and $\left\{y_{n}\right\}$ converging uniformly to $u^{*}$. Furthermore, we have the following iterative sequences

$$
\begin{aligned}
& x_{n+1}(t)=\mathbb{E}_{\frac{1}{2}, 1}(-4 \sqrt{t})+\int_{0}^{t}(t-s)^{-1 / 2} \mathbb{E}_{\frac{1}{2}, \frac{1}{2}}(-4 \sqrt{t-s})\left(1-x_{n}^{2}(s)+2 s+4 x_{n}(s)\right) \mathrm{d} s, t \in \mathrm{J}, \\
& y_{n+1}(t)=E_{\frac{1}{2}, 1}(-4 \sqrt{t})+\int_{0}^{t}(t-s)^{-1 / 2} \mathbb{E}_{\frac{1}{2}, \frac{1}{2}}(-4 \sqrt{t-s})\left(1-y_{n}^{2}(s)+2 s+4 y_{n}(s)\right) \mathrm{d} s, t \in \mathrm{J} .
\end{aligned}
$$

We notice that the sequences are obtained by solving a recurrence formula of the type $v_{n+1}=A v_{n}$, with $A$ a suitable integral operator and $v_{0}$ given. So, by a simple numerical procedure, it is not difficult to represent some iterates of the recurrence sequence. We plot in Figure 1 the four first iterates of each sequence. We point out that the unique solution is lying within $x_{3}$ and $y_{3}$ which gives us a good approximation of such a solution. 


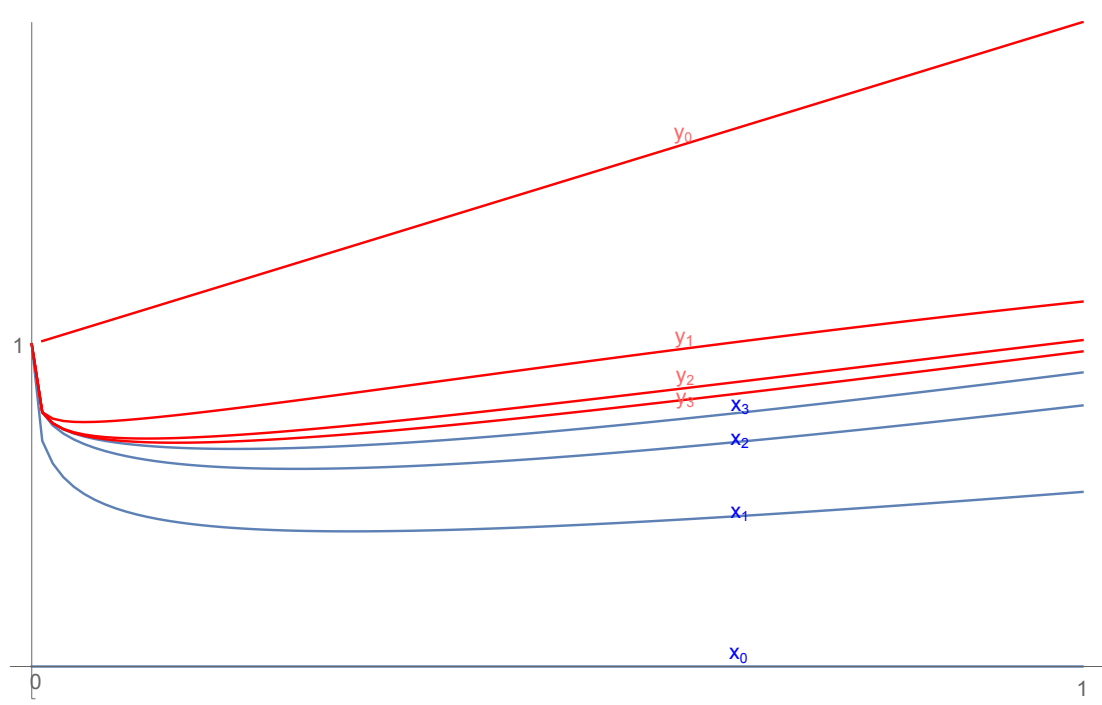

Figure 1. First four iterates for problem (20).

\section{Conclusions}

In previous sections, we have presented the existence and uniqueness of extremal solutions to a Cauchy problem with $\psi$-Caputo fractional derivative. Moreover, some uniqueness results are obtained. The proof of the existence results is based on the monotone iterative technique combined with the method of upper and lower solutions. Moreover, an example is presented to illustrate the validity of our main results. Our results are not only new in the given configuration but also correspond to some new situations associated with the specific values of the parameters involved in the given problem.

Author Contributions: conceptualization, C.D., Z.B., M.B. and A.C.; methodology, C.D., Z.B., M.B. and A.C.; formal analysis, C.D., Z.B., M.B. and A.C.; investigation, C.D., Z.B., M.B. and A.C.; writing-original draft preparation, C.D., Z.B., M.B. and A.C.; writing-review and editing, C.D., Z.B., M.B. and A.C.; funding acquisition, A.C. All authors have read and agreed to the published version of the manuscript.

Funding: The fourth author is supported by the Agencia Estatal de Investigación (AEI) of Spain under grant MTM2016-75140-P, co-financed by the European Community fund FEDER. The fourth author is also supported by Xunta de Galicia, project ED431C 2019/02 (Spain).

Conflicts of Interest: The authors declare no conflict of interest.

\section{References}

1. Hilfer, R. Applications of Fractional Calculus in Physics; World Scientific: Singapore, 2000.

2. Oldham, K.B. Fractional differential equations in electrochemistry. Adv. Eng. Softw. 2010, 41, 9-12. [CrossRef]

3. Sabatier, J.; Agrawal, O.P.; Machado, J.A.T. Advances in Fractional Calculus-Theoretical Developments and Applications in Physics and Engineering; Springer: Dordrecht, The Netherlands, 2007.

4. Tarasov, V.E. Fractional Dynamics: Application of Fractional Calculus to Dynamics of Particles, Fields and Media; Springer Science \& Business Media: Berlin/Heidelberg, Germany, 2010.

5. Abbas, S.; Benchohra, M.; N'Gurékata, G.M. Topics in Fractional Differential Equations; Springer: New York, NY, USA, 2015.

6. Abbas, S.; Benchohra, M.; Graef, J.R.; Henderson, J. Implicit Fractional Differential and Integral Equations: Existence and Stability; De Gruyter: Berlin, Germany, 2018.

7. Abbas, S.; Benchohra, M.; N'Guŕékata, G.M. Advanced Fractional Differential and Integral Equations; Nova Sci. Publ.: New York, NY, USA, 2014.

8. Kilbas, A.A.; Srivastava, H.M.; Trujillo, J.J. Theory and Applications of Fractional Differential Equations; vol. 204 of North-Holland Mathematics Sudies; Elsevier Science B.V.: Amsterdam, The Netherlands, 2006.

9. Miller, K.S.; Ross, B. An Introduction to Fractional Calculus and Fractional Differential Equations; Wiley: New York, NY, USA, 1993.

10. Podlubny, I. Fractional Differential Equations; Academic Press: San Diego, CA, USA, 1999. 
11. Zhou, Y. Basic Theory of Fractional Differential Equations; World Scientific: Singapore, 2014.

12. Zhou, Y. Fractional Evolution Equations and Inclusions; Analysis and Control; Elsevier, Acad. Press: Cambridge, MA, USA, 2016.

13. Agarwal, R.P.; Benchohra, M.; Hamani, S. A survey onexistence results for boundary value problems of nonlinear fractional differential equations and inclusions. Acta Appl. Math. 2010, 109, 973-1033. [CrossRef]

14. Benchohra, M.; Graef, J.R.; Hamani, S. Existence results for boundary value problems with non-linear fractional differential equations. Appl. Anal. 2008, 87, 851-863. [CrossRef]

15. Benchohra, M.; Hamani, S.; Ntouyas, S.K. Boundary value problems for differential equations with fractional order and nonlocal conditions. Nonlinear Anal. 2009, 71, 2391-2396. [CrossRef]

16. Benchohra, M.; Lazreg, J.E. Existence results for nonlinear implicit fractional differential equations. Surv. Math. Appl. 2014, 9, 79-92.

17. Almeida, R. A Caputo fractional derivative of a function with respect to another function. Commun. Nonlinear Sci. 2017, 44, 460-481. [CrossRef]

18. Abdo, M.S.; Panchal, S.K.; Saeed, A.M. Fractional boundary value problem with $\psi$-Caputo fractional derivative. Proc. Math. Sci. 2019, 129, 14. [CrossRef]

19. Almeida, R. Fractional Differential Equations with Mixed Boundary Conditions. Bull. Malays. Math. Sci. Soc. 2019, 42, 1687-1697. [CrossRef]

20. Almeida, R.; Malinowska, A.B.; Monteiro, M.T.T. Fractional differential equations with a Caputo derivative with respect to a kernel function and their applications. Math. Meth. Appl. Sci. 2018, 41, 336-352. [CrossRef]

21. Almeida, R.; Malinowska, A.B.; Odzijewicz, T. Optimal Leader-Follower Control for the Fractional Opinion Formation Model. J. Optim. Theory Appl. 2019, 182, 1171-1185. [CrossRef]

22. Almeida, R.; Jleli, M.; Samet, B. A numerical study of fractional relaxation-oscillation equations involving భ-Caputo fractional derivative. Rev. R. Acad. Cienc. Exactas Fís. Nat. Ser. A Mat. RACSAM 2019, 113, 1873-1891. [CrossRef]

23. Samet, B.; Aydi, H. Lyapunov-type inequalities for an anti-periodic fractional boundary value problem involving $\psi$-Caputo fractional derivative. J. Inequal. Appl. 2018, 2018, 286. [CrossRef] [PubMed]

24. Abbas, S.; Benchohra, M.; Samet, B.; Zhou, Y. Coupled implicit Caputo fractional q-difference systems. Adv. Diff. Equ. 2019, 2019, 527. [CrossRef]

25. Abbas, S.; Benchohra, M.; Hamidi, N.; Henderson, J. Caputo-Hadamard fractional differential equations in Banach spaces. Fract. Calc. Appl. Anal. 2018, 21, 1027-1045. [CrossRef]

26. Abbas, S.; Benchohra, M.; Hamani, S.; Henderson, J. Upper and lower solutions method for Caputo-Hadamard fractional differential inclusions. Math. Morav. 2019, 23, 107-118. [CrossRef]

27. Aghajani, A.; Pourhadi, E.; Trujillo, J.J. Application of measure of noncompactness to a Cauchy problem for fractional differential equations in Banach spaces. Fract. Calc. Appl. Anal. 2013, 16, 962-977. [CrossRef]

28. Kucche, K.D.; Mali, A.D.; Sousa, J.V.C. On the nonlinear $\Psi-H i l f e r$ fractional differential equations. Comput. Appl. Math. 2019, 38, 25. [CrossRef]

29. Wu, G.C.; Zeng, D.Q.; Baleanu, D. Fractional impulsive differential equations: Exact solutions, integral equations and short memory case. Fract. Calc Appl. Anal. 2019, 22, 180-192. [CrossRef]

30. Wu, G.C.; Deng, Z.G.; Baleanu, D.; Zeng, D.Q. New variable order fractional chaotic systems for fast image encryption. Chaos 2019, 29, 11. [CrossRef]

31. Ali, S.; Shah, K.; Jarad, F. On stable iterative solutions for a class of boundary value problem of nonlinear fractional order differential equations. Math. Methods Appl. Sci. 2019, 42, 969-981. [CrossRef]

32. Al-Refai, M.; Ali Hajji, M. Monotone iterative sequences for nonlinear boundary value problems of fractional order. Nonlinear Anal. 2011, 74, 3531-3539. [CrossRef]

33. Chen, C.; Bohner, M.; Jia, B. Method of upper and lower solutions for nonlinear Caputo fractional difference equations and its applications. Fract. Calc. Appl. Anal. 2019, 22, 1307-1320. [CrossRef]

34. Dhaigude, D.; Rizqan, B. Existence and uniqueness of solutions of fractional differential equations with deviating arguments under integral boundary conditions. Kyungpook Math. J. 2019, 59, 191-202.

35. Fazli, H.; Sun, H.; Aghchi, S. Existence of extremal solutions of fractional Langevin equation involving nonlinear boundary conditions. Int. J. Comput. Math. 2020. [CrossRef]

36. Lin, X.; Zhao, Z. Iterative technique for a third-order differential equation with three-point nonlinear boundary value conditions. Electron. J. Qual. Theory Differ. Equ. 2016, 12, 10. [CrossRef] 
37. Ma, K.; Han, Z.; Sun, S. Existence and uniqueness of solutions for fractional $q$-difference Schrödinger equations. J. Appl. Math. Comput. 2020, 62, 611-620. [CrossRef]

38. Mao, J.; Zhao, Z.; Wang, C. The unique iterative positive solution of fractional boundary value problem with q-difference. Appl. Math. Lett. 2020, 100, 106002. [CrossRef]

39. Meng, S.; Cui, Y. The extremal solution to conformable fractional differential equations involving integral boundary condition. Mathematics 2019, 7, 186. [CrossRef]

40. Wang, G.; Sudsutad, W.; Zhang, L.; Tariboon, J. Monotone iterative technique for a nonlinear fractional q-difference equation of Caputo type. Adv. Diff. Equ. 2016, 2016, 211. [CrossRef]

41. Yang, W. Monotone iterative technique for a coupled system of nonlinear Hadamard fractional differential equations. J. Appl. Math. Comput. 2019, 59, 585-596. [CrossRef]

42. Zhang, S. Monotone iterative method for initial value problem involving Riemann-Liouville fractional derivatives. Nonlinear Anal. 2009, 71, 2087-2093. [CrossRef]

43. Gorenflo, R.; Kilbas, A.A.; Mainardi, F.; Rogosin, S.V. Mittag-Leffler Functions, Related Topics and Applications; Springer: New York, NY, USA, 2014.

44. Diethelm, K.; Ford, N.J. Analysis of fractional differential equations. J. Math. Anal. Appl. 2002, 265, $229-248$. [CrossRef]

45. Nieto, J.J. Maximum principles for fractional differential equations derived from Mittag-Leffler functions. Appl. Math. Lett. 2010, 23, 1248-1251. [CrossRef]

46. Royden, H.L. Real Analysis, 3rd ed.; Macmillan Publishing Company: New York, NY, USA, 1988.

(C) 2020 by the authors. Licensee MDPI, Basel, Switzerland. This article is an open access article distributed under the terms and conditions of the Creative Commons Attribution (CC BY) license (http:/ / creativecommons.org/licenses/by/4.0/). 